\title{
Iterative approximation of attractive points of further generalized hybrid mappings in Hadamard spaces
}

\author{
Asawathep Cuntavepanit ${ }^{1}$ and Withun Phuengrattana $2^{2^{*}}$
}

\section{"Correspondence:}

withun_ph@yahoo.com

${ }^{2}$ Department of Mathematics,

Faculty of Science and Technology,

Nakhon Pathom Rajabhat

University, Nakhon Pathom,

Thailand

Full list of author information is

available at the end of the article

\begin{abstract}
In this paper, we study the class of further generalized hybrid mappings due to Khan (Fixed Point Theory Appl. 2018:8, 2018) in the setting of Hadamard spaces. We prove a demiclosed principle for such mappings in Hadamard spaces. Furthermore, we also prove the $\Delta$-convergence of the sequence generated by the S-iteration process for finding attractive points of further generalized hybrid mappings in Hadamard spaces satisfying the $(\mathbb{S})$ property and the $\left(\overline{Q_{4}}\right)$ condition. Moreover, we provide a numerical example to illustrate the convergence behavior of the studied iteration and numerically compare the convergence of the studied iteration scheme with the existing schemes. Our results extend some known results which appeared in the literature.
\end{abstract}

MSC: $47 \mathrm{H} 09 ; 47 \mathrm{H} 10$

Keywords: Attractive point; Further generalized hybrid mappings; S-iteration; $\Delta$-convergence; Hadamard spaces

\section{Introduction}

In 2011, Takahashi and Takeuchi [2] introduced the concept of attractive points for nonlinear mappings in a Hilbert space: Let $H$ be a Hilbert space and $C$ be a nonempty subset of $H$. Let $T$ be a mapping from $C$ into $H$. Let $A(T)$ denote the set of all attractive points of $T$, i.e.,

$$
A(T)=\{z \in H:\|T x-z\| \leq\|x-z\|, \forall x \in C\} .
$$

In 2012, Takahashi et al. [3] introduced the class of normally generalized hybrid mappings in a Hilbert space.

Definition 1.1 A mapping $T: C \rightarrow H$ is called normally generalized hybrid if there exist $\alpha, \beta, \gamma, \delta \in \mathbb{R}$ such that

(i) $\alpha+\beta+\gamma+\delta \geq 0$

(ii) $\alpha+\beta>0$ or $\alpha+\gamma>0$; and

(iii) $\alpha\|T x-T y\|^{2}+\beta\|x-T y\|^{2}+\gamma\|T x-y\|^{2}+\delta\|x-y\|^{2} \leq 0, \forall x, y \in C$.

Such a mapping $T$ can be called an $(\alpha, \beta, \gamma, \delta)$-normally generalized hybrid mapping.

\section{Springer}


They also proved the weak convergence theorem of Mann type for normally generalized hybrid mappings in real Hilbert spaces without convexity assumption on the domain of mappings. To be more precise, they obtained the following result.

Theorem 1.2 Let $H$ be a real Hilbert space and $C$ be a nonempty convex subset of $H$. Let $T: C \rightarrow C$ be a normally generalized hybrid mapping. Assume that $A(T) \neq \emptyset$. For $x_{1} \in C$, the sequence $\left\{x_{n}\right\}$ generated by

$$
x_{n+1}=\left(1-\alpha_{n}\right) x_{n}+\alpha_{n} T x_{n}, \quad n \in \mathbb{N},
$$

where $\left\{\alpha_{n}\right\}$ is a sequence in $[0,1]$ such that $\liminf _{n \rightarrow \infty} \alpha_{n}\left(1-\alpha_{n}\right)>0$. Then the sequence $\left\{x_{n}\right\}$ converges weakly to a point $z \in A(T)$. Moreover, $z=\lim _{n \rightarrow \infty} P_{A(T)} x_{n}$, where $P_{A(T)}$ is a projection of $H$ onto $A(T)$.

In 2015, Kaewkhao et al. [4] extended the results of Takahashi et al. [3] from Hilbert spaces to Hadamard spaces.

In 2018, Khan [1] gave the concept of further generalized mappings (see Definition 1.3 below) which constitutes a generalization of normally generalized hybrid mappings due to Takahashi et al. [3] (see Definition 1.1 above).

Definition 1.3 ([1]) A mapping $T: C \rightarrow H$ is called further generalized hybrid if there exist $\alpha, \beta, \gamma, \delta, \epsilon \in \mathbb{R}$ such that

(i) $\alpha+\beta+\gamma+\delta \geq 0, \epsilon \geq 0$;

(ii) $\alpha+\beta>0$ or $\alpha+\gamma>0$; and

(iii) $\alpha\|T x-T y\|^{2}+\beta\|x-T y\|^{2}+\gamma\|T x-y\|^{2}+\delta\|x-y\|^{2}+\epsilon\|x-T x\|^{2} \leq 0, \forall x, y \in C$.

Obviously, by above definitions, further generalized hybrid is a generalization of normally generalized hybrid when $\epsilon=0$. It is noteworthy that it contains the class of generalized hybrid, quasi-nonexpansive mappings, quasi-contractive mappings and contractive mappings.

Recently, Khan [1] obtained a weak convergence theorem of Picard-Mann hybrid iterative process [5] for further generalized hybrid mappings in real Hilbert spaces without convexity assumption on the domain of mappings. The iterative process of Khan [1] is faster than Mann, Ishikawa and S-iteration process of Agarwal et al. [6] as shown by him in [5]. However, his results are in a Hilbert space and we want to have some results in Hadamard spaces. Note that no results are available at the moment for further generalized hybrid mappings even for Mann iterative process in Hadamard spaces. We further note that $\mathrm{S}$-iteration process is also faster than Mann and Ishikawa iteration processes (but not Picard-Mann hybrid).

Motivated by the above works, we define a class of further generalized hybrid mappings and prove the demiclosed principle for such mapping in Hadamard spaces. Furthermore, we also obtain a $\Delta$-convergence theorem of $\mathrm{S}$-iteration process for further generalized hybrid mappings in Hadamard spaces satisfying the $(\mathbb{S})$ property and the $\left(\overline{Q_{4}}\right)$ condition. Finally, we provide a numerical example to illustrate the convergence behavior of the Siteration and numerically compare the convergence of the S-iteration schemes with the existing schemes. 


\section{Methods}

The paper is organized as follows. Section 3 contains the preliminaries, including definitions and lemmas with corresponding references that will be used in the sequel. Section 4 contains the main result of the paper. In Sect. 5, we provide a numerical example to illustrate the convergence behavior of the $\mathrm{S}$-iteration and numerically compare the convergence of the S-iteration schemes with the existing schemes.

\section{Preliminaries}

Let $(X, d)$ be a metric space. A geodesic from $x$ to $y$ is a map $\gamma$ from the closed interval $[0, d(x, y)] \subset \mathbb{R}$ to $X$ such that $\gamma(0)=x, \gamma(d(x, y))=y$ and $d\left(\gamma\left(t_{1}\right), \gamma\left(t_{2}\right)\right)=\left|t_{1}-t_{2}\right|$ for all $t_{1}, t_{2} \in[0, d(x, y)]$. The image of $\gamma$ is called a geodesic (or metric) segment joining $x$ and $y$. When it is unique, this geodesic segment is denoted by $[x, y]$. The space $X$ is said to be a geodesic metric space if every two points of $X$ are joined by a geodesic, and $X$ is said to be uniquely geodesic metric space if there is exactly one geodesic joining $x$ and $y$ for each $x, y \in X$. A subset $C$ of $X$ is said to be convex, if for any two points $x, y \in C$, the geodesic joining $x$ and $y$ is contained in $C$.

Let $X$ be a uniquely geodesic metric space. For each $x, y \in X$ and for each $\alpha \in[0,1]$, there exists a unique point $z \in[x, y]$ such that $d(x, z)=(1-\alpha) d(x, y)$ and $d(y, z)=\alpha d(x, y)$. We denote the unique point $z$ by $\alpha x \oplus(1-\alpha) y$.

Lemma 3.1 ([7]) Let X be a uniquely geodesic metric space. The following are equivalent:

(i) $X$ is a $C A T(0)$ space.

(ii) $X$ satisfies the $(C N)$ inequality: If $x, y \in X$ and $\frac{x \oplus y}{2}$ is the midpoint of $x$ and $y$, then

$$
d\left(z, \frac{x \oplus y}{2}\right)^{2} \leq \frac{1}{2} d(z, x)^{2}+\frac{1}{2} d(z, y)^{2}-\frac{1}{4} d(x, y)^{2}, \quad \text { for all } z \in X .
$$

Lemma 3.2 ([7, 8]) Let $X$ be a $C A T(0)$ space, $x, y, z \in X$ and $\lambda \in[0,1]$. Then

(i) $d(z, \lambda x \oplus(1-\lambda) y) \leq \lambda d(z, x)+(1-\lambda) d(z, y)$;

(ii) $d(z, \lambda x \oplus(1-\lambda) y)^{2} \leq \lambda d(z, x)^{2}+(1-\lambda) d(z, y)^{2}-\lambda(1-\lambda) d(x, y)^{2}$.

A complete CAT(0) space is called an Hadamard space.

It is well known that any complete, simply connected Riemannian manifold having nonpositive sectional curvature is an Hadamard space. Other examples include Euclidean spaces $\mathbb{E}^{2}$, Hilbert spaces, the Hilbert ball [9], hyperbolic spaces [10], R-trees [11], and many others. The fixed point theory in Hadamard spaces was first studied by Kirk [12] in 2003. Since then many authors have published papers on the existence and convergence of fixed points for nonlinear mappings in such spaces (e.g., see [13, 14]).

The notion of the asymptotic center can be introduced in the general setting of an Hadamard space $X$ as follows: Let $\left\{x_{n}\right\}$ be a bounded sequence in $X$. For $x \in X$, we define a mapping $r\left(\cdot,\left\{x_{n}\right\}\right): X \rightarrow[0, \infty)$ by

$$
r\left(x,\left\{x_{n}\right\}\right)=\limsup _{n \rightarrow \infty} d\left(x, x_{n}\right)
$$

The asymptotic radius of $\left\{x_{n}\right\}$ is given by

$$
r\left(\left\{x_{n}\right\}\right)=\inf \left\{r\left(x,\left\{x_{n}\right\}\right): x \in X\right\},
$$


and the asymptotic center of $\left\{x_{n}\right\}$ is the set

$$
A\left(\left\{x_{n}\right\}\right)=\left\{x \in X: r\left(x,\left\{x_{n}\right\}\right)=r\left(\left\{x_{n}\right\}\right)\right\} .
$$

For another way of describing the asymptotic center, see [13]. It is known (see [15]) that in an Hadamard space, the asymptotic center $A\left(\left\{x_{n}\right\}\right)$ consists of exactly one point.

We now give the definition and collect some basic properties of the $\Delta$-convergence which will be used in the sequel.

Definition 3.3 ([16]) A sequence $\left\{x_{n}\right\}$ in an Hadamard space $X$ is said to $\Delta$-converge to $x \in X$ if $x$ is the unique asymptotic center of $\left\{u_{n}\right\}$ for every subsequence $\left\{u_{n}\right\}$ of $\left\{x_{n}\right\}$. In this case, we write $\Delta-\lim _{n \rightarrow \infty} x_{n}=x$ and call $x$ the $\Delta$-limit of $\left\{x_{n}\right\}$.

Lemma 3.4 ([16]) Every bounded sequence in an Hadamard space has a $\Delta$-convergent subsequence.

Lemma 3.5 ([17]) Let $C$ be a nonempty closed convex subset of an Hadamard space X. If $\left\{x_{n}\right\}$ is a bounded sequence in $C$, then the asymptotic center of $\left\{x_{n}\right\}$ is in $C$.

Lemma 3.6 ([8]) Let $\left\{x_{n}\right\}$ be a sequence in an Hadamard space $X$ with $A\left(\left\{x_{n}\right\}\right)=\{x\}$. If $\left\{u_{n}\right\}$ is a subsequence of $\left\{x_{n}\right\}$ with $A\left(\left\{u_{n}\right\}\right)=\{u\}$ and $\left\{d\left(x_{n}, u\right)\right\}$ converges, then $x=u$.

In 2008, Berg and Nikolaev [18] introduced the concept of quasilinearization in an Hadamard space $X$ as follows:

Denote a pair $(a, b) \in X \times X$ by $\overrightarrow{a b}$ and call it a vector. The quasilinearization is a map $\langle\cdot, \cdot\rangle:(X \times X) \times(X \times X) \rightarrow \mathbb{R}$ defined by

$$
\langle\overrightarrow{a b}, \overrightarrow{c d}\rangle=\frac{1}{2}\left(d(a, d)^{2}+d(b, c)^{2}-d(a, c)^{2}-d(b, d)^{2}\right)
$$

for any $a, b, c, d \in X$.

We say that $X$ satisfies the Cauchy-Schwarz inequality if

$$
\langle\overrightarrow{a b}, \overrightarrow{c d}\rangle \leq d(a, b) d(c, d)
$$

for any $a, b, c, d \in X$. It is known that a geodesically connected metric space is a $\operatorname{CAT}(0)$ space if and only if it satisfies the Cauchy-Schwarz inequality; see [18].

Consider the map $\Theta: \mathbb{R} \times X \times X \rightarrow C(X ; \mathbb{R})$ defined by

$$
\Theta(t, a, b)(x)=t\langle\overrightarrow{a b}, \overrightarrow{a x}\rangle
$$

for all $x \in X$, where $C(X ; \mathbb{R})$ is the space of all continuous real-valued functions on $X$. Then the Cauchy-Schwarz inequality implies that $\Theta(t, a, b)$ is the Lipschitz function with the Lipschitz seminorm $L(\Theta(t, a, b))=t d(a, b)$, where $t \in \mathbb{R}, a, b \in X$ and $L(\phi)=\sup \left\{\frac{\phi(x)-\phi(y)}{d(x, y)}\right.$ : $x, y \in X, x \neq y$ \} is the Lipschitz seminorm for any function $\phi: X \rightarrow \mathbb{R}$.

In 2010, Kakavandi and Amini [19] defined a pseudometric $D$ on $\mathbb{R} \times X \times X$ by

$$
D((t, a, b),(s, u, v))=L(\Theta(t, a, b)-\Theta(s, u, v)) .
$$


For an Hadamard space $X$, it is obtained that $D((t, a, b),(s, u, v))=0$ if and only if $t\langle\overrightarrow{a b}, \overrightarrow{x y}\rangle=$ $s\langle\overrightarrow{u v}, \overrightarrow{x y}\rangle$ for all $x, y \in X$. Then, $D$ can impose an equivalent relation on $\mathbb{R} \times X \times X$, where the equivalence class of $(t, a, b)$ is

$$
[t \overrightarrow{a b}]=\{s \overrightarrow{u v}: t\langle\overrightarrow{a b}, \overrightarrow{x y}\rangle=s\langle\overrightarrow{u v}, \overrightarrow{x y}\rangle\}
$$

The set $X^{*}=\{[t \overrightarrow{a b}]:(t, a, b) \in \mathbb{R} \times X \times X\}$ is a metric space with metric $D$, which is called the dual metric space of $X$.

In 2013, Kakavandi [20] introduced the concept of (S) property for an Hadamard space as follows.

Definition 3.7 An Hadamard space $X$ satisfies the (S) property if for any $(x, y) \in X \times X$ there exists a point $y_{x} \in X$ such that $[\overrightarrow{x y}]=\left[\overrightarrow{y_{x}}\right]$.

Moreover, Kakavandi also proved the characterization of $\Delta$-convergence for Hadamard spaces satisfying the $(\mathbb{S})$ property as follows.

Lemma 3.8 Let $X$ be an Hadamard space, $\left\{x_{n}\right\}$ be a bounded sequence in $X$ and $x \in X$. If $X$ satisfies the $(\mathbb{S})$ property, then $\Delta-\lim _{n \rightarrow \infty} x_{n}=x$ if and only if $\lim _{n \rightarrow \infty}\left\langle\overrightarrow{x x_{n}}, \overrightarrow{x y}\right\rangle=0$ for all $y \in X$.

In 2008, Kirk and Panyanak [16] introduced a geometric condition on Hadamard spaces called the $\left(Q_{4}\right)$ condition as follows.

Definition 3.9 An Hadamard space $X$ is said to satisfy the $\left(Q_{4}\right)$ condition if for all $x, y, p, q \in X$,

$$
d(p, x)<d(x, q) \quad \text { and } \quad d(p, y)<d(y, q) \quad \text { imply } \quad d(p, m)<d(m, q), \quad \forall m \in[x, y] .
$$

In 2013, Kakavandi [20] modified the $\left(Q_{4}\right)$ condition as follows.

Definition 3.10 An Hadamard space $X$ is said to satisfy the $\left(\overline{Q_{4}}\right)$ condition if for any $x, y, p, q \in X$,

$$
d(p, x) \leq d(x, q) \quad \text { and } \quad d(p, y) \leq d(y, q) \quad \text { imply } \quad d(p, m) \leq d(m, q), \quad \forall m \in[x, y] .
$$

We can see that Hilbert spaces and every Hadamard space of constant curvature satisfy the $\left(\overline{Q_{4}}\right)$ condition. Anyway, since $\left(\overline{Q_{4}}\right)$ implies $\left(Q_{4}\right)$, there are some Hadamard spaces that do not satisfy such a condition. The following results were obtained by Kaewkhao et al. [4].

Lemma 3.11 Let $X$ be an Hadamard space satisfying the $\left(\overline{Q_{4}}\right)$ condition. Let $C$ be a nonempty subset of $X$. Then, for any mapping $T: C \rightarrow X, A(T)$ is closed and convex.

Lemma 3.12 Let $X$ be an Hadamard space and $C$ be a closed convex subset of X. Let $x \in X$ and $y \in C$. Then $y=P_{C} x$ if and only if $\langle\overrightarrow{x y}, \overrightarrow{y z}\rangle \geq 0$ for all $z \in C$. 
We recall the concept of Banach limit, which plays a major role in our results. Let $\mu$ be a continuous linear functional on $l^{\infty}$, the Banach space of bounded real sequences with supremum norm, and $\left(x_{1}, x_{2}, \ldots\right) \in l^{\infty}$. We write $\mu_{n}\left(x_{n}\right)$ instead of $\mu\left(\left(x_{1}, x_{2}, \ldots\right)\right)$. We call $\mu$ a Banach limit if $\mu$ satisfies $\|\mu\|=\mu(1,1, \ldots)=1$ and $\mu_{n}\left(x_{n}\right)=\mu_{n}\left(x_{n+1}\right)$ for each $\left(x_{1}, x_{2}, \ldots\right) \in l^{\infty}$. For a Banach limit $\mu$, we know that

$$
\liminf _{n \rightarrow \infty} x_{n} \leq \mu_{n}\left(x_{n}\right) \leq \limsup _{n \rightarrow \infty} x_{n}
$$

for all $\left(x_{1}, x_{2}, \ldots\right) \in l^{\infty}$. So if $\left(x_{1}, x_{2}, \ldots\right) \in l^{\infty}$ with $\lim _{n \rightarrow \infty} x_{n}=c$, then $\mu_{n}\left(x_{n}\right)=c$; see [21] for more details.

We also need the following lemmas due to Kaewkhao et al. [4].

Lemma 3.13 Let $C$ be a nonempty subset of an Hadamard space X. Let $\left\{x_{n}\right\}$ be a bounded sequence in $C$ and $T: C \rightarrow C$ be a mapping such that $\lim _{n \rightarrow \infty} d\left(x_{n}, T x_{n}\right)=0$. Then

(i) the sequences $\left\{d\left(x_{n}, y\right)\right\}$ and $\left\{d\left(T x_{n}, y\right)\right\}$ are bounded for all $y \in C$;

(ii) $\mu_{n} d\left(x_{n}, y\right)=\mu_{n} d\left(T x_{n}, y\right)$ for any Banach limit $\mu_{n}$ on $l^{\infty}$.

Lemma 3.14 Let $X$ be an Hadamard space and $C$ be a closed convex subset of $X$. Let $\left\{x_{n}\right\}$ be a bounded sequence in $X$.If $d\left(x_{n+1}, z\right) \leq d\left(x_{n}, z\right)$ for all $z \in C$, then $\lim _{n \rightarrow \infty} P_{C} x_{n}=z_{0} \in C$, where $P_{C}$ is the metric projection from $X$ onto $C$.

\section{Results and discussion}

In this section, we prove $\Delta$-convergence theorems of S-iteration to the set of attractive points of further generalized hybrid mappings in Hadamard spaces satisfying the ( $\mathbb{S}$ ) property and the $\left(\overline{Q_{4}}\right)$ condition. We first consider the notion of the set of attractive points for any mapping $T: C \rightarrow X$, where $X$ is an Hadamard space and $C$ is a nonempty subset of $X$ defined as

$$
A(T)=\{z \in X: d(T x, z) \leq d(x, z), \forall x \in C\} .
$$

Moreover, in Hadamard spaces, a further generalized hybrid mapping is defined analogously to Definition 1.3 as follows.

Definition 4.1 A mapping $T: C \rightarrow X$ is called further generalized hybrid if there exist $\alpha, \beta, \gamma, \delta, \epsilon \in \mathbb{R}$ such that

(i) $\alpha+\beta+\gamma+\delta \geq 0, \epsilon \geq 0$;

(ii) $\alpha+\beta>0$ or $\alpha+\gamma>0$; and

(iii) $\alpha d(T x, T y)^{2}+\beta d(x, T y)^{2}+\gamma d(T x, y)^{2}+\delta d(x, y)^{2}+\epsilon d(x, T x)^{2} \leq 0, \forall x, y \in C$.

The following lemma is a demiclosedness principle for a further generalized hybrid mapping in an Hadamard space.

Lemma 4.2 Let $X$ be an Hadamard space $X$ satisfying the $(\mathbb{S})$ property. Let $C$ be a nonempty subset of $X$ and let $T: C \rightarrow C$ be a further generalized hybrid mapping. Let $\left\{x_{n}\right\}$ be a bounded sequence in $C$ such that $\lim _{n \rightarrow \infty} d\left(x_{n}, T x_{n}\right)=0$ and $\Delta-\lim _{n \rightarrow \infty} x_{n}=z$. Then $z \in A(T)$. 
Proof Since $T$ is a further generalized hybrid mapping,

$$
\alpha d(T x, T y)^{2}+\beta d(x, T y)^{2}+\gamma d(T x, y)^{2}+\delta d(x, y)^{2}+\epsilon d(x, T x)^{2} \leq 0,
$$

for all $x, y \in C$. Lemma 3.13(i) implies that $\left\{d\left(x_{n}, y\right)\right\}$ and $\left\{d\left(T x_{n}, y\right)\right\}$ are bounded for all $y \in C$.

If $\alpha+\beta>0$, then

$$
\alpha d\left(T x_{n}, T y\right)^{2}+\beta d\left(x_{n}, T y\right)^{2}+\gamma d\left(T x_{n}, y\right)^{2}+\delta d\left(x_{n}, y\right)^{2}+\epsilon d\left(x_{n}, T x_{n}\right)^{2} \leq 0 .
$$

We apply a Banach limit $\mu$ to both sides of this inequality. Thus, we have

$$
\alpha \mu_{n} d\left(T x_{n}, T y\right)^{2}+\beta \mu_{n} d\left(x_{n}, T y\right)^{2}+\gamma \mu_{n} d\left(T x_{n}, y\right)^{2}+\delta \mu_{n} d\left(x_{n}, y\right)^{2}+\epsilon \mu_{n} d\left(x_{n}, T x_{n}\right)^{2} \leq 0 .
$$

Since $\mu_{n} d\left(x_{n}, T x_{n}\right)^{2}=0$, we get

$$
(\alpha+\beta) \mu_{n} d\left(x_{n}, T y\right)^{2}+(\gamma+\delta) \mu_{n} d\left(x_{n}, y\right)^{2} \leq 0
$$

for all $y \in C$. Since $\alpha+\beta+\gamma+\delta \geq 0$ and $\alpha+\beta>0$, we have

$$
\mu_{n} d\left(x_{n}, T y\right)^{2} \leq \frac{-(\gamma+\delta)}{\alpha+\beta} \mu_{n} d\left(x_{n}, y\right)^{2} \leq \mu_{n} d\left(x_{n}, y\right)^{2} .
$$

If $\alpha+\gamma>0$, then

$$
\alpha d\left(T y, T x_{n}\right)^{2}+\beta d\left(y, T x_{n}\right)^{2}+\gamma d\left(T y, x_{n}\right)^{2}+\delta d\left(y, x_{n}\right)^{2}+\epsilon d(y, T y)^{2} \leq 0 .
$$

We apply a Banach limit $\mu$ to both sides of this inequality. Thus, by $\mu_{n} d\left(x_{n}, T x_{n}\right)^{2}=0$, we have

$$
(\alpha+\gamma) \mu_{n} d\left(T y, x_{n}\right)^{2}+\epsilon \mu_{n} d(y, T y)^{2} \leq-(\beta+\delta) \mu_{n} d\left(y, x_{n}\right)^{2} .
$$

It follows from $\epsilon \geq 0$ that

$$
\begin{aligned}
(\alpha+\gamma) \mu_{n} d\left(T y, x_{n}\right)^{2} & \leq(\alpha+\gamma) \mu_{n} d\left(T y, x_{n}\right)^{2}+\epsilon \mu_{n} d(y, T y)^{2} \\
& \leq-(\beta+\delta) \mu_{n} d\left(y, x_{n}\right)^{2}
\end{aligned}
$$

for all $y \in C$. Since $\alpha+\beta+\gamma+\delta \geq 0$ and $\alpha+\gamma>0$, we have

$$
\mu_{n} d\left(T y, x_{n}\right)^{2} \leq \frac{-(\beta+\delta)}{\alpha+\gamma} \mu_{n} d\left(y, x_{n}\right)^{2} \leq \mu_{n} d\left(y, x_{n}\right)^{2} .
$$

Therefore,

$$
\mu_{n} d\left(x_{n}, T y\right)^{2} \leq \mu_{n} d\left(x_{n}, y\right)^{2}
$$


for all $y \in C$. Furthermore, Lemma 3.8 and $\Delta-\lim _{n \rightarrow \infty} x_{n}=z$ imply that

$$
\lim _{n \rightarrow \infty}\left\langle\overrightarrow{z x_{n}}, \overrightarrow{z y}\right\rangle=0
$$

This implies that

$$
\lim _{n \rightarrow \infty}\left(d\left(x_{n}, z\right)^{2}-d\left(x_{n}, y\right)^{2}+d(z, y)^{2}\right)=0
$$

for all $y \in X$. Thus, we have

$$
\mu_{n}\left(d\left(x_{n}, z\right)^{2}-d\left(x_{n}, y\right)^{2}+d(z, y)^{2}\right)=0
$$

for all $y \in X$. By (2), we obtain that

$$
-\mu_{n} d\left(x_{n}, y\right)^{2} \leq-\mu_{n} d\left(x_{n}, T y\right)^{2} .
$$

By adding $\mu_{n}\left(d\left(x_{n}, z\right)^{2}+d(z, y)^{2}+d(z, T y)^{2}\right)$ to both sides of the above inequality, we get

$$
\begin{aligned}
- & \mu_{n} d\left(x_{n}, y\right)^{2}+\mu_{n}\left(d\left(x_{n}, z\right)^{2}+d(z, y)^{2}+d(z, T y)^{2}\right) \\
& \leq-\mu_{n} d\left(x_{n}, T y\right)^{2}+\mu_{n}\left(d\left(x_{n}, z\right)^{2}+d(z, y)^{2}+d(z, T y)^{2}\right) .
\end{aligned}
$$

Then we have

$$
\begin{aligned}
& d(z, T y)^{2}+\mu_{n}\left(d\left(x_{n}, z\right)^{2}-d\left(x_{n}, y\right)^{2}+d(z, y)^{2}\right) \\
& \quad \leq d(z, y)^{2}+\mu_{n}\left(d\left(x_{n}, z\right)^{2}-d\left(x_{n}, T y\right)^{2}+d(z, T y)^{2}\right) .
\end{aligned}
$$

This implies by (3) that $d(z, T y) \leq d(z, y)$, and hence $z \in A(T)$.

In what follows we get a $\Delta$-convergence theorem for a further generalized hybrid mapping in an Hadamard space.

Theorem 4.3 Let X be an Hadamard space satisfying the $(\mathbb{S})$ property and the $\left(\overline{Q_{4}}\right)$ condition. Let $C$ be a nonempty convex subset of $X$ and $T: C \rightarrow C$ be a further generalized hybrid mapping with $A(T) \neq \emptyset$. Let $\left\{\alpha_{n}\right\},\left\{\beta_{n}\right\}$ be sequences of real numbers such that $0<a \leq \alpha_{n}$, $\beta_{n} \leq b<1$ for all $n \in \mathbb{N}$ and for some $a, b$. Suppose that $\left\{x_{n}\right\}$ is the sequence generated by the S-iteration process: let $x_{1}=x \in C$ and

$$
\left\{\begin{array}{l}
y_{n}=\left(1-\beta_{n}\right) x_{n} \oplus \beta_{n} T x_{n} \\
x_{n+1}=\left(1-\alpha_{n}\right) T x_{n} \oplus \alpha_{n} T y_{n}, \quad \forall n \in \mathbb{N} .
\end{array}\right.
$$

Then the sequence $\left\{x_{n}\right\} \Delta$-converges to an element $v \in A(T)$, where $v=\lim _{n \rightarrow \infty} P_{A(T)} x_{n}$ and $P_{A(T)}$ is the metric projection from $X$ onto $A(T)$. 
Proof Step 1. We will show that $\lim _{n \rightarrow \infty} d\left(u, x_{n}\right)$ exists for all $u \in A(T)$.

Let $u \in A(T)$. Then, by (4), we have

$$
\begin{aligned}
d\left(u, x_{n+1}\right) & =d\left(u,\left(1-\alpha_{n}\right) T x_{n} \oplus \alpha_{n} T y_{n}\right) \\
& \leq\left(1-\alpha_{n}\right) d\left(u, T x_{n}\right)+\alpha_{n} d\left(u, T y_{n}\right) \\
& \leq\left(1-\alpha_{n}\right) d\left(u, x_{n}\right)+\alpha_{n} d\left(u, y_{n}\right) .
\end{aligned}
$$

Also, we get

$$
\begin{aligned}
d\left(u, y_{n}\right) & =d\left(u,\left(1-\beta_{n}\right) x_{n} \oplus \beta_{n} T x_{n}\right) \\
& \leq\left(1-\beta_{n}\right) d\left(u, x_{n}\right)+\beta_{n} d\left(u, T x_{n}\right) \\
& \leq d\left(u, x_{n}\right) .
\end{aligned}
$$

By (5) and (6), we obtain

$$
d\left(u, x_{n+1}\right) \leq d\left(u, x_{n}\right) .
$$

This shows that the sequence $\left\{d\left(u, x_{n}\right)\right\}$ is decreasing and bounded below. Therefore, $\lim _{n \rightarrow \infty} d\left(u, x_{n}\right)$ exists for any $u \in A(T)$ and so $\left\{x_{n}\right\}$ must be bounded.

Step 2. We will show that $\lim _{n \rightarrow \infty} d\left(x_{n}, T x_{n}\right)=0$.

Let

$$
\lim _{n \rightarrow \infty} d\left(u, x_{n}\right)=c
$$

By (5), we have

$$
d\left(u, x_{n+1}\right) \leq\left(1-\alpha_{n}\right) d\left(u, x_{n}\right)+\alpha_{n} d\left(u, y_{n}\right)
$$

This gives

$$
\alpha_{n} d\left(u, x_{n}\right) \leq d\left(u, x_{n}\right)+\alpha_{n} d\left(u, y_{n}\right)-d\left(u, x_{n+1}\right)
$$

and so

$$
\begin{aligned}
d\left(u, x_{n}\right) & \leq d\left(u, y_{n}\right)+\frac{1}{\alpha_{n}}\left(d\left(u, x_{n}\right)-d\left(u, x_{n+1}\right)\right) \\
& \leq d\left(u, y_{n}\right)+\frac{1}{a}\left(d\left(u, x_{n}\right)-d\left(u, x_{n+1}\right)\right) .
\end{aligned}
$$

Thus, we have

$$
\liminf _{n \rightarrow \infty} d\left(u, x_{n}\right) \leq \liminf _{n \rightarrow \infty}\left(d\left(u, y_{n}\right)+\frac{1}{a}\left(d\left(u, x_{n}\right)-d\left(u, x_{n+1}\right)\right)\right) .
$$

Since $\left\{d\left(u, y_{n}\right)\right\}$ is bounded and $\lim _{n \rightarrow \infty} d\left(u, x_{n}\right)$ exists, we obtain that

$$
\liminf _{n \rightarrow \infty} d\left(u, x_{n}\right) \leq \liminf _{n \rightarrow \infty} d\left(u, y_{n}\right)+\lim _{n \rightarrow \infty}\left(\frac{1}{a}\left(d\left(u, x_{n}\right)-d\left(u, x_{n+1}\right)\right)\right)
$$


So we get

$$
c \leq \liminf _{n \rightarrow \infty} d\left(u, y_{n}\right)
$$

By (6), we have

$$
\limsup _{n \rightarrow \infty} d\left(u, y_{n}\right) \leq \liminf _{n \rightarrow \infty} d\left(u, x_{n}\right)=c
$$

Therefore,

$$
\lim _{n \rightarrow \infty} d\left(u, y_{n}\right)=c
$$

By Lemma 3.2, we obtain

$$
\begin{aligned}
d\left(u, y_{n}\right)^{2} & =d\left(u,\left(1-\beta_{n}\right) x_{n} \oplus \beta_{n} T x_{n}\right)^{2} \\
& \leq\left(1-\beta_{n}\right) d\left(u, x_{n}\right)^{2}+\beta_{n} d\left(u, T x_{n}\right)^{2}-\beta_{n}\left(1-\beta_{n}\right) d\left(x_{n}, T x_{n}\right)^{2} \\
& \leq\left(1-\beta_{n}\right) d\left(u, x_{n}\right)^{2}+\beta_{n} d\left(u, x_{n}\right)^{2}-\beta_{n}\left(1-\beta_{n}\right) d\left(x_{n}, T x_{n}\right)^{2} \\
& =d\left(u, x_{n}\right)^{2}-\beta_{n}\left(1-\beta_{n}\right) d\left(x_{n}, T x_{n}\right)^{2} .
\end{aligned}
$$

Thus, we have

$$
\begin{aligned}
d\left(x_{n}, T x_{n}\right)^{2} & \leq \frac{1}{\beta_{n}\left(1-\beta_{n}\right)}\left(d\left(u, x_{n}\right)^{2}-d\left(u, y_{n}\right)^{2}\right) \\
& \leq \frac{1}{a(1-b)}\left(d\left(u, x_{n}\right)^{2}-d\left(u, y_{n}\right)^{2}\right)
\end{aligned}
$$

Using (7) and (8), we can conclude that

$$
\lim _{n \rightarrow \infty} d\left(x_{n}, T x_{n}\right)=0
$$

Step 3. We will show that the sequence $\left\{x_{n}\right\} \Delta$-converges to an element in $A(T)$. Indeed, since $\left\{x_{n}\right\}$ is bounded, we can assume that $A\left(\left\{x_{n}\right\}\right)=\{v\}$ for some $v \in X$. It is sufficient to show that $A\left(\left\{x_{n_{k}}\right\}\right)=\{v\}$ for any subsequence $\left\{x_{n_{k}}\right\}$ of $\left\{x_{n}\right\}$. Let $\left\{x_{n_{k}}\right\}$ be a subsequence of $\left\{x_{n}\right\}$ with $A\left(\left\{x_{n_{k}}\right\}\right)=\{w\}$. Since $\left\{x_{n_{k}}\right\}$ is bounded, there exists a subsequence $\left\{x_{n_{j}}\right\}$ of $\left\{x_{n_{k}}\right\}$ such that $\left\{x_{n_{j}}\right\} \Delta$-converges to $z$ for some $z \in X$. By (9) and Lemma 4.2, we have $z \in A(T)$ and hence $\lim _{n \rightarrow \infty} d\left(z, x_{n}\right)$ exists. If $z \neq w$, then it follows from the uniqueness of asymptotic center that

$$
\begin{aligned}
\lim _{n \rightarrow \infty} d\left(z, x_{n}\right) & =\limsup _{j \rightarrow \infty} d\left(z, x_{n_{j}}\right) \\
& <\limsup _{j \rightarrow \infty} d\left(w, x_{n_{j}}\right) \\
& \leq \limsup _{k \rightarrow \infty} d\left(w, x_{n_{k}}\right) \\
& <\limsup _{k \rightarrow \infty} d\left(z, x_{n_{k}}\right)
\end{aligned}
$$




$$
=\lim _{n \rightarrow \infty} d\left(z, x_{n}\right)
$$

which is a contradiction. Therefore, $w=z \in A(T)$. Suppose that $v \neq w$. Then

$$
\begin{aligned}
\lim _{n \rightarrow \infty} d\left(w, x_{n}\right) & =\limsup _{k \rightarrow \infty} d\left(w, x_{n_{k}}\right) \\
& \leq \limsup _{k \rightarrow \infty} d\left(v, x_{n_{k}}\right) \\
& \leq \limsup _{n \rightarrow \infty} d\left(v, x_{n}\right) \\
& <\limsup _{n \rightarrow \infty} d\left(w, x_{n}\right) \\
& =\lim _{n \rightarrow \infty} d\left(w, x_{n}\right) .
\end{aligned}
$$

This leads to a contradiction, and hence $v=w \in A(T)$. Therefore, $\left\{x_{n}\right\} \Delta$-converges to an element $v \in A(T)$.

Step 4. We will show that $v=\lim _{n \rightarrow \infty} P_{A(T)} x_{n}$. We can conclude from Step 1 that $d\left(x_{n+1}, z\right) \leq d\left(x_{n}, z\right)$ for all $z \in A(T)$ and $n \in \mathbb{N}$. Furthermore, we obtain from Lemma 3.14 that

$$
\lim _{n \rightarrow \infty} P_{A(T)} x_{n}=p
$$

for some $p \in A(T)$. From Step 1, we have that $\lim _{n \rightarrow \infty} d\left(p, x_{n}\right)$ exists. Since

$$
d\left(x_{n+1}, P_{A(T)} x_{n+1}\right) \leq d\left(x_{n+1}, P_{A(T)} x_{n}\right) \leq d\left(x_{n}, P_{A(T)} x_{n}\right)
$$

for all $n \in \mathbb{N}$, we get that $\lim _{n \rightarrow \infty} d\left(x_{n}, P_{A(T)} x_{n}\right)$ exists. By the triangle inequality, we have

$$
\begin{aligned}
d\left(x_{n}, p\right) & \leq d\left(x_{n}, P_{A(T)} x_{n}\right)+d\left(P_{A(T)} x_{n}, p\right) \\
& \leq d\left(x_{n}, p\right)+d\left(P_{A(T)} x_{n}, p\right) .
\end{aligned}
$$

Since $\lim _{n \rightarrow \infty} d\left(P_{A(T)} x_{n}, p\right)=0$, we have

$$
\lim _{n \rightarrow \infty} d\left(x_{n}, p\right) \leq \lim _{n \rightarrow \infty} d\left(x_{n}, P_{A(T)} x_{n}\right) \leq \lim _{n \rightarrow \infty} d\left(x_{n}, p\right) .
$$

This implies

$$
\lim _{n \rightarrow \infty} d\left(x_{n}, p\right)=\lim _{n \rightarrow \infty} d\left(x_{n}, P_{A(T)} x_{n}\right)
$$

Moreover, since $\left\{x_{n}\right\} \Delta$-converges to $v \in A(T)$, we can apply Lemma 3.8 to conclude that

$$
0=\lim _{n \rightarrow \infty}\left\langle\overrightarrow{\left\langle x_{n}\right.}, \overrightarrow{v z}\right\rangle=\lim _{n \rightarrow \infty} \frac{1}{2}\left(d\left(x_{n}, v\right)^{2}+d(v, z)^{2}-d\left(x_{n}, z\right)^{2}\right)
$$

for all $z \in X$. This implies

$$
-d^{2}(v, p)=\lim _{n \rightarrow \infty}\left(d\left(x_{n}, v\right)^{2}-d\left(x_{n}, p\right)^{2}\right) .
$$


So, by (10), (11) and Lemma 3.12, we have

$$
\begin{aligned}
-2 d^{2}(v, p) & =\lim _{n \rightarrow \infty}\left(d\left(x_{n}, v\right)^{2}-d\left(x_{n}, p\right)^{2}-d(v, p)^{2}\right) \\
& =\lim _{n \rightarrow \infty}\left(d\left(x_{n}, v\right)^{2}-d\left(x_{n}, P_{A(T)} x_{n}\right)^{2}-d\left(v, P_{A(T)} x_{n}\right)^{2}\right) \\
& =\lim _{n \rightarrow \infty}\left\langle\overrightarrow{x_{n} P_{A(T)} x_{n}}, \widehat{P_{A(T)} x_{n} v}\right\rangle \\
& \geq 0 .
\end{aligned}
$$

This implies that $d(v, p)^{2} \leq 0$, and hence $v=p$. Therefore, $\left\{x_{n}\right\} \Delta$-converges to $v$, where $v=\lim _{n \rightarrow \infty} P_{A(T)} x_{n}$.

Remark 4.4 Theorem 4.3 extends and improves the results of Kaewkhao et al. [4] from a normally generalized hybrid mapping to a further generalized hybrid mapping. In fact, we present the $\mathrm{S}$-iteration process for solving the attractive point problem of further generalized hybrid mappings in Hadamard spaces.

It is known that a Hilbert space satisfies both the $(\mathbb{S})$ property and the $\left(\overline{Q_{4}}\right)$ condition. Furthermore, $\Delta$-convergence and weak convergence are the same in a Hilbert space. Thus, we have the following theorem.

Theorem 4.5 Let $X$ be a Hilbert space. Let $C$ be a nonempty convex subset of $X$ and $T$ : $C \rightarrow C$ be a further generalized hybrid mapping with $A(T) \neq \emptyset$. Let $\left\{\alpha_{n}\right\},\left\{\beta_{n}\right\}$ be sequences of real numbers such that $0<a \leq \alpha_{n}, \beta_{n} \leq b<1$ for all $n \in \mathbb{N}$ and for some $a, b$. Suppose that $\left\{x_{n}\right\}$ is the sequence generated by the S-iteration process: let $x_{1}=x \in C$ and

$$
\left\{\begin{array}{l}
y_{n}=\left(1-\beta_{n}\right) x_{n}+\beta_{n} T x_{n}, \\
x_{n+1}=\left(1-\alpha_{n}\right) T x_{n}+\alpha_{n} T y_{n}, \quad \forall n \in \mathbb{N} .
\end{array}\right.
$$

Then the sequence $\left\{x_{n}\right\}$ converges weakly to an element $v \in A(T)$, where $v=\lim _{n \rightarrow \infty} P_{A(T)} x_{n}$ and $P_{A(T)}$ is the metric projection from $X$ onto $A(T)$.

Moreover, the following example shows that there is an Hadamard space satisfying both the $(\mathbb{S})$ property and the $\left(\overline{Q_{4}}\right)$ condition, which is not a Hilbert space.

Example 4.6 ([4]) Consider $\mathcal{H}=\left\{(x, y) \in \mathbb{R}^{2}: y^{2}-x^{2}=1\right.$ and $\left.y>0\right\}$. Let $d$ be a metric defined by the function $d: \mathcal{H} \times \mathcal{H} \rightarrow \mathbb{R}$ that assigns to each pair of vectors $u=\left(u_{1}, u_{2}\right)$ and $v=\left(v_{1}, v_{2}\right)$ the unique nonnegative number $d(u, v) \geq 0$ such that

$$
\cosh d(u, v)=u_{2} v_{2}-u_{1} v_{1}
$$

It is known that, in general, the metric space $(\mathcal{H}, d)$ is an Hadamard space and also a onedimensional hyperbolic space viewed from a hyperboloid model (for more details, see [7]). Then $(\mathcal{H}, d)$ satisfies the $(\mathbb{S})$ property and the $\left(\overline{Q_{4}}\right)$ condition.

Remark 4.7 Theorem 4.5 extends and improves the results of Takahashi et al. [3] from a normally generalized hybrid mapping to a further generalized hybrid mapping. In fact, we 
present the S-iteration process for solving the attractive point problem of further generalized hybrid mappings in Hilbert spaces.

\section{Numerical example for the main result}

In this section, we give a numerical example supporting our main results and compare the convergence of the studied method (4) with the Mann and Ishikawa iterations.

Recall that the Mann iteration [22] is defined by $u_{1} \in C$ and

$$
u_{n+1}=\left(1-\alpha_{n}\right) u_{n}+\alpha_{n} T u_{n}, \quad n \in \mathbb{N},
$$

where $\left\{\alpha_{n}\right\}$ is a sequence in $(0,1)$. The Ishikawa iteration [23] is defined by $z_{1} \in C$ and

$$
\left\{\begin{array}{l}
w_{n}=\left(1-\beta_{n}\right) z_{n}+\beta_{n} T z_{n}, \\
z_{n+1}=\left(1-\alpha_{n}\right) z_{n}+\alpha_{n} T w_{n}, \quad \forall n \in \mathbb{N},
\end{array}\right.
$$

where $\left\{\alpha_{n}\right\}$ and $\left\{\beta_{n}\right\}$ are sequences in $(0,1)$.

Example 5.1 Let $X=\mathbb{R}$ be a usual metric space with the metric $d$, which is also an Hadamard space, and $C=(-1,1)$. We see that $C$ is a convex subset of $X$. Define a mapping $T: C \rightarrow C$ by

$$
T x= \begin{cases}\frac{1-x}{2}, & x \in(-1,0] \\ \frac{x+1}{2}, & x \in(0,1) .\end{cases}
$$

for all $x \in C$. It is easy to see that $T$ is a further generalized hybrid mapping with $\alpha=2, \beta=$ $\gamma=-1, \delta=\epsilon=0$ and $A(T)=[1, \infty)$. Let $\alpha_{n}=\frac{4 n}{5 n+7}$ and $\beta_{n}=\frac{n+3}{10 n+1}$ for all $n \in \mathbb{N}$. Let $\left\{x_{n}\right\}$ be a sequence generated by $S$-iteration (4), $\left\{u_{n}\right\}$ be a sequence generated by Mann iteration (12) and $\left\{z_{n}\right\}$ be a sequence generated by Ishikawa iteration (13). The numerical experiments of all iterations for approximating the attractive point 1 , where $1=\lim _{n \rightarrow \infty} P_{A(T)} x_{n}$, and convergence of $\left\{x_{n}\right\},\left\{u_{n}\right\}$ and $\left\{z_{n}\right\}$ are given in Tables 1 and 2 .

From Tables 1 and 2, we see that both $\left\{x_{n}\right\},\left\{u_{n}\right\}$ and $\left\{z_{n}\right\}$ converge to $1 \in A(T)$ and observe that $\left|x_{n}-1\right| \leq\left|u_{n}-1\right|$ and $\left|x_{n}-1\right| \leq\left|z_{n}-1\right|$, so the sequence $\left\{x_{n}\right\}$ generated by $S$ iteration converges faster than both $\left\{u_{n}\right\}$ generated by Mann iteration and $\left\{z_{n}\right\}$ generated by Ishikawa iteration.

\section{Conclusions}

The results presented in this paper modify, extend and improve the corresponding results of Takahashi et al. [3] and Kaewkhao et al. [4], and others. The main aim of this paper is to prove the demiclosed principle for further generalized hybrid mapping and the $\Delta$-convergence of the sequence generated by the $S$-iteration process for finding attractive points of such mappings in Hadamard spaces satisfying the (S) property and the $\left(\overline{Q_{4}}\right)$ condition. We also provide a numerical example to illustrate and support our results at the end. 
Table 1 Iterates of S-iteration, Mann iteration, and Ishikawa iteration for $x_{1}=u_{1}=z_{1}=-0.5$

\begin{tabular}{|c|c|c|c|c|c|c|}
\hline \multirow[t]{2}{*}{$\bar{n}$} & \multicolumn{2}{|l|}{ S-iteration } & \multicolumn{2}{|c|}{ Mann iteration } & \multicolumn{2}{|c|}{ Ishikawa iteration } \\
\hline & $x_{n}$ & $\left|x_{n}-x_{n-1}\right|$ & $u_{n}$ & $\left|u_{n}-u_{n-1}\right|$ & $z_{n}$ & $\left|z_{n}-z_{n-1}\right|$ \\
\hline 1 & -0.5000000 & - & -0.5000000 & - & -0.5000000 & - \\
\hline 2 & 0.6742424 & $1.1742 \mathrm{e}+00$ & -0.0833333 & $4.1667 \mathrm{e}-01$ & -0.1590909 & $3.4091 \mathrm{e}-01$ \\
\hline 3 & 0.8462461 & $1.7200 \mathrm{e}-01$ & 0.2107843 & $2.9412 \mathrm{e}-01$ & 0.1550166 & $3.1411 \mathrm{e}-01$ \\
\hline 4 & 0.9271811 & $8.0935 \mathrm{e}-02$ & 0.4260250 & $2.1524 \mathrm{e}-01$ & 0.4077682 & $2.5275 \mathrm{e}-01$ \\
\hline 5 & 0.9654324 & $3.8251 \mathrm{e}-02$ & 0.5960916 & $1.7007 \mathrm{e}-01$ & 0.5982240 & $1.9046 \mathrm{e}-01$ \\
\hline & . & : & . & : & & : \\
\hline 16 & 0.9999899 & $1.1023 e-05$ & 0.9962619 & $2.1566 \mathrm{e}-03$ & 0.9974837 & $1.5930 \mathrm{e}-03$ \\
\hline 17 & 0.9999952 & $5.2699 \mathrm{e}-06$ & 0.9976368 & $1.3749 \mathrm{e}-03$ & 0.9984638 & $9.8015 e-04$ \\
\hline 18 & 0.9999977 & $2.5201 \mathrm{e}-06$ & 0.9985102 & $8.7334 \mathrm{e}-04$ & 0.9990648 & $6.0091 \mathrm{e}-04$ \\
\hline 19 & 0.9999989 & $1.2054 \mathrm{e}-06$ & 0.9990631 & $5.5292 \mathrm{e}-04$ & 0.9994320 & $3.6724 \mathrm{e}-04$ \\
\hline 20 & 0.9999995 & $5.7667 \mathrm{e}-07$ & 0.9994121 & $3.4904 \mathrm{e}-04$ & 0.9996558 & $2.2380 \mathrm{e}-04$ \\
\hline
\end{tabular}

Table 2 Iterates of S-iteration, Mann iteration, and Ishikawa iteration for $x_{1}=u_{1}=z_{1}=0.4$

\begin{tabular}{|c|c|c|c|c|c|c|}
\hline \multirow[t]{2}{*}{$n$} & \multicolumn{2}{|l|}{ S-iteration } & \multicolumn{2}{|c|}{ Mann iteration } & \multicolumn{2}{|c|}{ Ishikawa iteration } \\
\hline & $x_{n}$ & $\left|x_{n}-x_{n-1}\right|$ & $u_{n}$ & $\left|u_{n}-u_{n-1}\right|$ & $z_{n}$ & $\left|z_{n}-z_{n-1}\right|$ \\
\hline 1 & 0.4000000 & - & 0.4000000 & - & 0.4000000 & - \\
\hline 2 & 0.7181818 & $3.1818 \mathrm{e}-01$ & 0.5000000 & $1.0000 \mathrm{e}-01$ & 0.5181818 & $1.1818 \mathrm{e}-01$ \\
\hline 3 & 0.8669850 & $1.4880 \mathrm{e}-01$ & 0.6176471 & $1.1765 \mathrm{e}-01$ & 0.6450471 & $1.2687 \mathrm{e}-01$ \\
\hline 4 & 0.9370031 & $7.0018 \mathrm{e}-02$ & 0.7219251 & $1.0428 \mathrm{e}-01$ & 0.7512207 & $1.0617 \mathrm{e}-01$ \\
\hline 5 & 0.9700950 & $3.3092 \mathrm{e}-02$ & 0.8043177 & $8.2393 e-02$ & 0.8312256 & $8.0005 e-02$ \\
\hline & $\vdots$ & $\vdots$ & $\vdots$ & $\vdots$ & $\vdots$ & $\vdots$ \\
\hline 16 & 0.9999913 & $9.5358 \mathrm{e}-06$ & 0.9981890 & $1.0448 \mathrm{e}-03$ & 0.9989430 & $6.6918 \mathrm{e}-04$ \\
\hline 17 & 0.9999958 & $4.5591 \mathrm{e}-06$ & 0.9988551 & $6.6612 \mathrm{e}-04$ & 0.9993547 & $4.1173 \mathrm{e}-04$ \\
\hline 18 & 0.9999980 & $2.1802 \mathrm{e}-06$ & 0.9992782 & $4.2311 \mathrm{e}-04$ & 0.9996071 & $2.5242 \mathrm{e}-04$ \\
\hline 19 & 0.9999990 & $1.0428 \mathrm{e}-06$ & 0.9995461 & $2.6788 \mathrm{e}-04$ & 0.9997614 & $1.5427 \mathrm{e}-04$ \\
\hline 20 & 0.9999995 & $4.9889 \mathrm{e}-07$ & 0.9997152 & $1.6910 \mathrm{e}-04$ & 0.9998554 & $9.4011 \mathrm{e}-05$ \\
\hline
\end{tabular}

\section{Acknowledgements}

The authors appreciate the support of their institutes.

\section{Funding}

The first author was supported by Mahidol University Kanchanaburi Campus, Kanchanaburi, Thailand.

\section{Abbreviations}

Not applicable.

Availability of data and materials

Data sharing not applicable to this article as no data sets were generated or analyzed during the current study.

\section{Competing interests}

The authors declare that they have no competing interests.

\section{Authors' contributions}

All authors contributed equally and significantly in writing this paper. All authors read and approved the final manuscript.

\section{Author details}

'Division of Sciences and Liberal Arts, Mahidol University Kanchanaburi Campus, Kanchanaburi, Thailand. ${ }^{2}$ Department of Mathematics, Faculty of Science and Technology, Nakhon Pathom Rajabhat University, Nakhon Pathom, Thailand.

\section{Publisher's Note}

Springer Nature remains neutral with regard to jurisdictional claims in published maps and institutional affiliations.

Received: 7 June 2018 Accepted: 4 January 2019 Published online: 28 January 2019

\section{References}

1. Khan, S.H.: Iterative approximation of common attractive points of further generalized hybrid mappings. Fixed Point Theory Appl. 2018, 8 (2018) 
2. Takahashi, W., Takeuchi, Y.: Nonlinear ergodic theorem without convexity for generalized hybrid mappings in a Hilbert space. J. Nonlinear Convex Anal. 12, 399-406 (2011)

3. Takahashi, W., Wong, N.-C., Yao, J.-C.: Attractive point and weak convergence theorems for new generalized hybrid mappings in Hilbert spaces. J. Nonlinear Convex Anal. 13, 745-757 (2012)

4. Kaewkhao, A., Inthakon, W., Kunwai, K.: Attractive points and convergence theorems for normally generalized hybrid mappings in CAT(0) spaces. Fixed Point Theory Appl. 2015, 96 (2015)

5. Khan, S.H.: A Picard-Mann hybrid iterative process. Fixed Point Theory Appl. 2013, 69 (2013)

6. Agarwal, R.P., O'Regan, D., Sahu, D.R.: Iterative construction of fixed points of nearly asymptotically nonexpansive mappings. J. Nonlinear Convex Anal. 8(1), 61-79 (2007)

7. Bridson, M., Haefliger, A.: Metric Spaces of Non-Positive Curvature. Springer, Berlin (1999)

8. Dhompongsa, S., Panyanak, B.: On $\Delta$-convergence theorems in CAT(0) spaces. Comput. Math. Appl. 56, 2572-2579 (2008)

9. Goebel, K., Reich, S.: Uniform Convexity, Hyperbolic Geometry, and Nonexpansive Mappings. Dekker, New York (1984)

10. Kohlenbach, U.: Some logical metatheorems with applications in functional analysis. Trans. Am. Math. Soc. 357, 89-128 (2015)

11. Tits, J.: A Theorem of Lie-Kolchin for Trees, Contributions to Algebra: A Collection of Papers Dedicated to Ellis Kolchin. Academic Press, New York (1977)

12. Kirk, W.A.: Geodesic geometry and fixed point theory. In: Seminar of Mathematical Analysis, Malaga/Seville, 2002/2003. Colecc. Abierta, vol. 64, pp. 195-225. Univ. Sevilla Secr. Publ., Seville (2003)

13. Reich, S., Salinas, Z.: Weak convergence of infinite products of operators in Hadamard spaces. Rend. Circ. Mat. Palermo 65, 55-71 (2016)

14. Reich, S., Shafrir, I.: Nonexpansive iterations in hyperbolic spaces. Nonlinear Anal. 15, 537-558 (1990)

15. Dhompongsa, S., Kirk, W.A., Sims, B.: Fixed points of uniformly Lipschitzian mappings. Nonlinear Anal. 65, 762-772 (2006)

16. Kirk, W.A., Panyanak, B.: A concept of convergence in geodesic spaces. Nonlinear Anal. 68, 3689-3696 (2008)

17. Dhompongsa, S., Kirk, W.A., Panyanak, B.: Nonexpansive set-valued mappings in metric and Banach spaces. J. Nonlinear Convex Anal. 8, 35-45 (2007)

18. Berg, I.D., Nikolaev, I.G.: Quasilinearization and curvature of Alexandrov spaces. Geom. Dedic. 133, 195-218 (2008)

19. Kakavandi, B.A., Amini, M.: Duality and subdifferential for convex functions on complete CAT(0) metric spaces. Nonlinear Anal. 73, 3450-3455 (2010)

20. Kakavandi, B.A.: Weak topologies in complete CAT(0) metric spaces. Proc. Am. Math. Soc. 141, 1029-1039 (2013)

21. Takahashi, W: Nonlinear Function Analysis. Yokahama Publishers, Yokahama (2000)

22. Mann, W.R.: Mean value methods in iteration. Proc. Am. Math. Soc. 4, 506-510 (1953)

23. Ishikawa, S.: Fixed points by a new iteration method. Proc. Am. Math. Soc. 44, 147-150 (1974)

\section{Submit your manuscript to a SpringerOpen ${ }^{\circ}$ journal and benefit from:}

- Convenient online submission

- Rigorous peer review

- Open access: articles freely available online

- High visibility within the field

- Retaining the copyright to your article

Submit your next manuscript at $\gg$ springeropen.com 